\title{
Study of interplanetary parameters, polar cap potential, and polar cap index during quiet event and high intensity long duration continuous AE activities (HILDCAAs)
}

\author{
B. Adhikari ${ }^{1}$, N. Sapkota ${ }^{1}$, B. Bhattarai ${ }^{1}$, R. K. Mishra ${ }^{1}$, S. Bohara ${ }^{1}$, N. P. Chapagain ${ }^{2}$, and B. Regmi ${ }^{1}$
}

Received 26 October 2017; accepted 12 January 2018; published 22 January 2018.

We observed the interplanetary datasets, polar cap potential (PCV), three different types of High Intensity Long Duration Continuous AE Activities (HILDCAAs) and polar cap index (PCI) during geomagnetically quiet period. On each event, we examine the interplanetary electric field $(E y)$, polar cap potential (PCV), polar cap index (PCI) and westward auroral electrojet (AL) indices. We found little perturbations in Ey during the quiet event, but significant perturbations during HILDCAAs. In particular, non-storm HILDCAA showed more perturbations in Ey compared to the other two HILDCAAs. Due to sporadic energy pumping into the magnetosphere, $E y$ was perturbed even after the non-storm HILDCAA. From CWT analysis, we found highest power intensities to have periodicity of more than 190 minutes for quiet event, non-storm HILDCAA and CIR-preceded HILDCAA. However, the magnitude of the higher power intensity was different: 11 units for PCV and PCI in quiet, 9 and 14 units respectively for PCV and PCI in non-storm HILDCAA, 15 units for PCV and PCI in CIR-preceded HILDCAA, and 23 and 14 units for PCV and PCI during ICME-preceded HILDCAA. PCV and PCI clearly showed that higher power intensities are found in higher timescales. In contrast, lower and middle power intensities are found across all timescales. KEYWORDS: Geomagnetic disturbances; Polar cap potential; HILDCAAs; Wavelet analysis.

Citation: Adhikari, B., N. Sapkota, B. Bhattarai, R. K. Mishra, S. Bohara, N. P. Chapagain, and B. Regmi (2018), Study of interplanetary parameters, polar cap potential, and polar cap index during quiet event and high intensity long duration continuous AE activities (HILDCAAs), Russ. J. Earth. Sci., 18, ES1001, doi:10.2205/2018ES000609.

\section{Introduction}

\footnotetext{
${ }^{1}$ Physics Department, St. Xavier's College, Maitighar, Kathmandu, Nepal

${ }^{2}$ Department of Physics, Patan Multiple Campus, Lalitpur, Nepal,
}

Copyright 2018 by the Geophysical Center RAS. http:/ /rjes.wdcb.ru/doi/2018ES000609-res.html
The fundamental concept of the magnetic field on the earth is based on the movement of a conducting fluid (mainly liquid iron) with sufficient speed in a suitable flow pattern. This flow of liquid iron engenders electric current and hence produces magnetic field. When charged metals are passing through this magnetic field, they produce 
electric currents of their own, and so the cycle continues. This self-propagating process is called as geodynamics [Mcelhinny, 1973]. The generation of magnetic field on the earth is also due to the electric currents in the ionosphere and magnetosphere. Even though its contribution for the magnetic field is weak, these currents cause geomagnetic disturbances. The root cause of geomagnetic disturbance are the electrojets in the ionosphere, caused by the fast moving solar energetic particles. The magnetic field associated with the electrojets perturbs the constant geomagnetic field and cause geomagnetic disturbances. Examples of geomagnetic disturbances are storms, substorms and High Intensity Long Duration Continuous AE Activities (HILDCAAs) HILDCAA events are defined by Tsurutani and Gonzalez, [1987] as events which last for longer than 2 days and have AE (Auroral Electrojet) peak values higher than $1000 \mathrm{nT}$ such that AE never reached below $200 \mathrm{nT}$ for period greater than 2 hours at a time. As the solar cycle approaches descending phase, increment takes place in the number of HILDCAA events and they persist for longer period of time. Moreover, AE index measures a higher auroral activity during this phase which maybe the result of long-term fast streams, originated from coronal holes, present in solar wind [Hajra et al.,2013. 2014. The component of Interplanetary magnetic field's $B z$ is expected to have a zero value but is found to have an average of $-0.70 \mathrm{nT}$ which indicates that during HILDCAA events, southward is the preferred direction (see HILDCAA* events between 1998 and 2007 and their related interplanetary magnetic field and plasma values, https://arxiv.org/abs/1612.03887). Majority of HILDCAA events are found to be associated with interplanetary Corotating Interaction Regions and High Speed Streamers (HSS). Few events are found to occur after passage of Interplanetary CMEs (ICMEs) [Hajra et al., 2013]. By the study of 214 ICMEs, Cane and Richardson, [2003] found that ICMEs had an average solar wind velocity of $454 \pm$ $6 \mathrm{~km} / \mathrm{s}$ which is less than the value observed for HILDCAA events. This helps to infer that majority of HILDCAA events have no relation with ICMEs. The HSS events produce relativistic electrons that can have dangerous impacts on space weather and orbiting spacecraft which is why some HILDCAA events (associated with HSS) may be associated with bad space weather [Hajra et al., 2014: Miyoshi et al., 2003].

Polar cap potential (PCV) is the difference between the extreme of the ionospheric electric convection potential i.e. $\mathrm{PCV}=\mathrm{PCV}_{\max }-\mathrm{PCV}_{\min }$ where $P C V_{\max } / \mathrm{PCV}_{\min }$ is the max./min. value of the convection electric potential in the highaltitude ionosphere [Shepherd, 2006]. It is considered to be an indicator for magnetospheric convection development.

The connection between the solar wind parameters and the polar cap potential is important to understand the coupling process between the solar wind and the magnetosphere and also the magnetosphere and the ionosphere [Boyle et al., 1997]. The network between the solar wind drivers and convection patterns in the Earth's polar ionosphere has been extensively studied [Hairston et al., 2005]. Among these solar wind drivers, the role of southward interplanetary magnetic field (IMF- $B z$ ) conditions have been understood very well [Boyle et al., 1997, Hairston et al., 2005, Weimer, 1996]. As the solar wind flows toward the earth, a crossmagnetospheric electric field is generated along the magnetopause. When the IMF- $B z$ is oriented southward, it reconnects directly with the Earth's magnetic field and a part of this electric field transfers to the polar ionosphere. This creates a potential difference in the ionosphere known as cross polar cap potential, which can be directly measured from low Earth orbiting satellites, radars, and ground magnetometers [Hairston et al., 2005: Kamide et al., 1981, Ruohoniemi and Baker, 1998]. For this study, one geomagnetically quiet period and three different types of HILDCAA events were selected: HILDCAA preceded by CIR storm, HILDCAA preceded by ICME and non-storm HILCAA. Through the analysis of such broad range of events, we expect to find some insights about the periodicities in polar cap potential and polar cap index during the HILDCAAs event and to compare them with those of the quiet event. These events were selected from the list of HILDCAAs events discussed by Hajra et al., [2013], based on the four criteria as suggested by Tsurutani and Gonzalez, [1987]. Interplanetary data sets used in this work are plasma speed $\left(V_{\mathrm{sw}}\right)$, and components of interplanetary magnetic field ( $B y$ and $B z$ ) and interplanetary dawn-dusk electric field $(E y)$. PCV was calculated as suggested by Kan and Lee, [1979] and Moon, [2012]. 


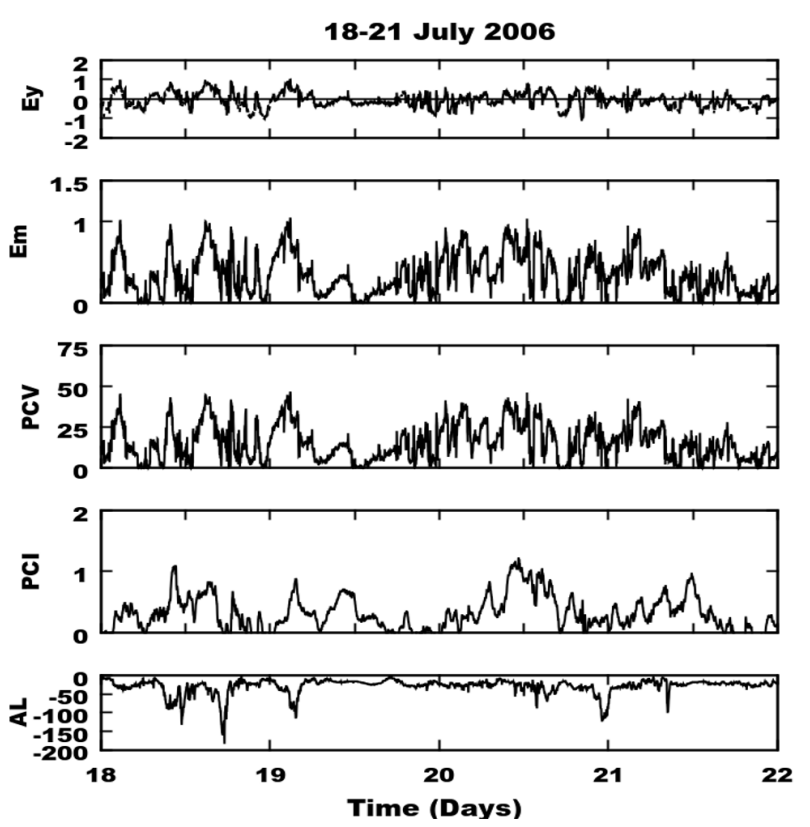

Figure 1. From top to bottom, (a) Interplanetary Electric Field $(E y$ in $\mathrm{mV} / \mathrm{m}$ ), (b) PCV (in $\mathrm{kV}$ ), (c) PCI (in $\mathrm{mV} / \mathrm{m}$ ) and (d) AL index (nT) during geomagnetically quiet period from 18th to 21st July 2006.

\section{Datasets}

The westward auroral electrojet (AL) index of 1-minute time resolution was used. It is downloaded from the OMNI website (http://cdaweb.gsfc.nasa.gov/). The PC index (1 min time resolution) used was also obtained from the same source. All these datasets of 1 minute's time resolution were also downloaded from the same source.

\section{Methodology}

Wavelet analysis (also termed as wavelet theory or simply wavelets) is the essence for signal processing especially non-stationary signals. A set of scaled and translated wavelets are generated from mother wavelet, which is the root of wavelet transform. A pioneer work about the introduction of wavelet analysis is given in [Lau and Weng, 1995]. A detailed explanation can be found in [Farge, 1992], [Daubechies, 1992], [Hubbard, 1998], [Domingues et al., 2005], and [An- toine et al., 2008. Wavelet transform acts as a bridge to transform data representation into its multilevel components [Daubechies, 1992].

\subsection{Continuous Wavelet Transform}

Over few decades, wavelet analysis has become an integral part for the analysis of non-stationary waves. Time-frequency analysis of non-stationary signals can be presented in a unique way using Continuous Wavelet Transform (CWT). CWT produces a time-scale map called scalogram for analysis of signals [Rioul and Vetterli, 1991]. Using CWT continuous time function can be transformed into wavelets [Grossmann and Morlet, 1983]. Wavelet coefficient $W(a, b)$ can be formulated as

$$
W(a, b)=\int f(t) \Psi^{*} \frac{t-b}{a} d t
$$

where $a$ and $b$ represent continuously varying dilation and translation parameters respectively. CWT paves a path for the evaluation of both low and high frequency signals [Adhikari, 2015]. The scale-parameter variation corresponds to contraction effect and dilation effect when $a<0$ and $a>0$ of mother wavelet respectively. This helps us analyze low or high frequencies, or long or short period features of signals. For details on CWT, please read [Domingues et al., 2005], and [Klausner et al., 2013].

\section{Result and Discussion}

\subsection{Events}

4.1.1. Quiet period (18th to 21st July 2006). Here, the $E y$ parameter has very little fluctuation which indicates very little disturbance in eastwest electric field. PCV is also strongly oriented towards eastward section giving $50 \mathrm{kV}$ as its peak value. Figure 1 denotes significant linkage between geomagnetic indices i.e. Polar Cap Index (PCI) and AL showing an inverse relation between them. The peak value for PCI is $1 \mathrm{mV} / \mathrm{m}$ and that of AL is $-200 \mathrm{nT}$. The value of AL indicates negligible intensity of electrojet in auroral ionosphere [Moon, 2012]. Electric currents in ionosphere cause geomagnetic daily variations [Stewart, 1982]. The layer of atmosphere $(60 \mathrm{~km}$ to 
19-24 April 2003
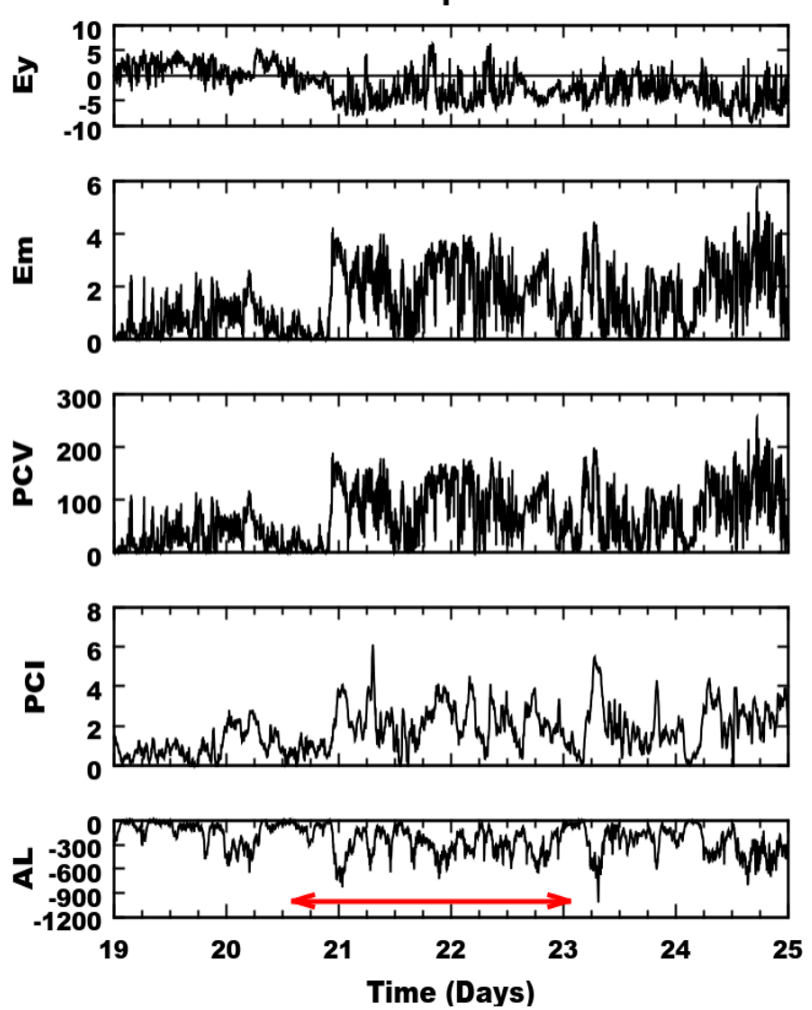

Figure 2. From top to bottom, (a) Interplanetary Electric Field $(E y$ in $\mathrm{mV} / \mathrm{m}$ ), (b) PCV (in $\mathrm{kV}$ ), (c) PCI $(\mathrm{mV} / \mathrm{m})$ and $(\mathrm{d})$ AL index $(\mathrm{nT})$ for a nonstorm HILDCAA event during 20-23 April, 2003. The purple horizontal double-headed arrow at the bottom of the plot indicates the HILDCAA interval.

$1000 \mathrm{~km}$ about Earth) undergoes ionization because of solar and cosmic radiations and is called ionosphere. Due to highly energetic cosmic rays, particles are photo ionized in this layer and thus, this layer is conducting. Solar Wind and thermal tide drive the partly ionized plasma that helps in conduction. This takes place in E-region that is about 50 to 90 miles of ionosphere. Compared to other events, the value of PCV and PCI are less in quiet day.

4.1.2.Non-storm HILDCAA (20-23 April,2003). Figure 2 demonstrates the combined plot of variation of $E y$ (in $\mathrm{mV} / \mathrm{m}$ ), PCV (in $\mathrm{kV}$ ), PCI (in $\mathrm{mV} / \mathrm{m}$ ) and AL (nT) for a particular HILDCAA event on 20-23 April, 2003. The figure reveals the high variability in the signal for Ey; however, during the HILDCAA period it mostly shows negative value which can also be referred as westward oscilla- tion of $E y$. It indicates the disturbances in eastwest electric field [Adhikari, 2015; Adhikari and Chapagain, 2016]. This shows fluctuation around $-5 \mathrm{mV} / \mathrm{m}$ throughout the HILDCAA event with peak of $-8 \mathrm{nV} / \mathrm{m}$. It is seen that PCI and $\mathrm{AL}$ have negative correlation between each other. It agrees with the result obtained by Moon, [2012]. Here, the average correlation coefficient between PCI and AL is -0.82 . The role of PCI and AL indices on polar cap potential can be investigated from [Adhikari and Chapagain, 2016]. The AL index provides idea about how intense the westward electrojet is in auroral ionosphere [Moon, 2012]. According to Echer et al., [2013], moderate forms of storm have average $E y=4 \mathrm{mV} / \mathrm{m}$. However, non-storm HILDCAA was also found to have higher value of $E y$ than expected [ $A d$ hikari and Chapagain, 2016]. High-intensity longduration continuous auroral electrojet activities (HILDCAAs) are magnetospheric and ionospheric events that mostly happen during the time of highspeed solar wind streams. The increment in Auroral Elecrojet (AE) are caused by sporadic magnetic reconnection between southward components of interplanetary Alfven wave fluctuations and magnetopause magnetic fields [Tsurutani et al., 2004]. The recovery phase will be long if there is a continuous injection of ions in the ring current. These injection processes were consistent with the variations in Alfven waves present in the magnetic field [Søraas et al., 2004].

\subsubsection{CIR preceding HILDCAA (12th to 15th}

February 2004). Interaction of distorted spirals formed by high speed streamers with slow streams forms CIR [Gosling and Pizzo, 1999]. At the time of declination of 11-year solar cycle, regions in solar surface called coronal holes, which have less density than all surrounding regions, are largest. During such phase, when the Sun rotates, the slow wind is caught up by the fast wind as they propagate away from their source i.e. the Sun. This creates compressed plasma at the boundary, increasing the density of the region of solar wind with slower velocity and decreasing the density in the region with fast solar wind [Alves et al., 2006]. This contact between the region with fast velocity and the one with slow velocity is called stream interface. Provided that the configuration of solar corona remains stable, pattern of such interaction regions keeps repeating as the Sun rotates and 

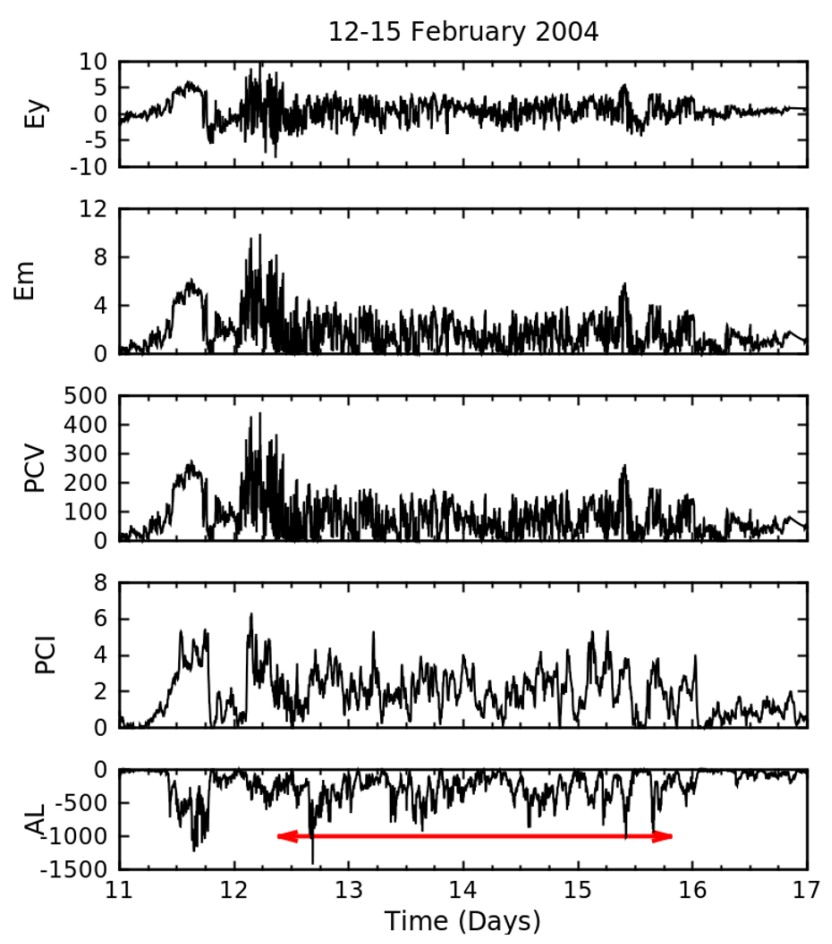

Figure 3. From top to bottom, (a) Interplanetary Electric Field $(E y$ in $\mathrm{mV} / \mathrm{m}$ ), (b) PCV (in $\mathrm{kV}$ ), (c) PCI $(\mathrm{mV} / \mathrm{m})$ and (d) AL index (nT) during HILDCAA (preceded by CIR-Storm) which occurred on 12th to 15th February 2004. The purple arrow indicates HILDCAA interval.

these regions are called Corotating Interaction Regions (CIRs) [Smith and Wolf, 1976]. As a result of CIR, regions of enhanced magnetic field strength and solar wind density are produced [Alves et al., 2006. These events play a vital role in geomagnetic disturbances created during solar minima [Gonzalez et al., 1999]. When the solar cycle is in its descending phase, the solar ejecta with high velocity occurs less often, which is why CIRs are formed more often [Feynman and $\mathrm{Gu}$, 1986].

Figure 3 shows CIR storms preceding HILDCAA event that occurred from 12th to 15th February 2004. The CIR storm is indicated by sharp fall in AL index on evening of 11th February 2004. The east-west electric field disturbances are significant few hours before the HILDCAA event, reaching a peak of $\sim 10 \mathrm{mV} / \mathrm{m}$. The average value of Ey during CIR storm is $\sim 4 \mathrm{mV} / \mathrm{m}$. According to Echer et al., [2013], the average value of $E y$ required in case of moderate storms is 4 $\mathrm{mV} / \mathrm{m}$ which is close to the Ey value of observed event. According to Alves et al., [2006], the aver-

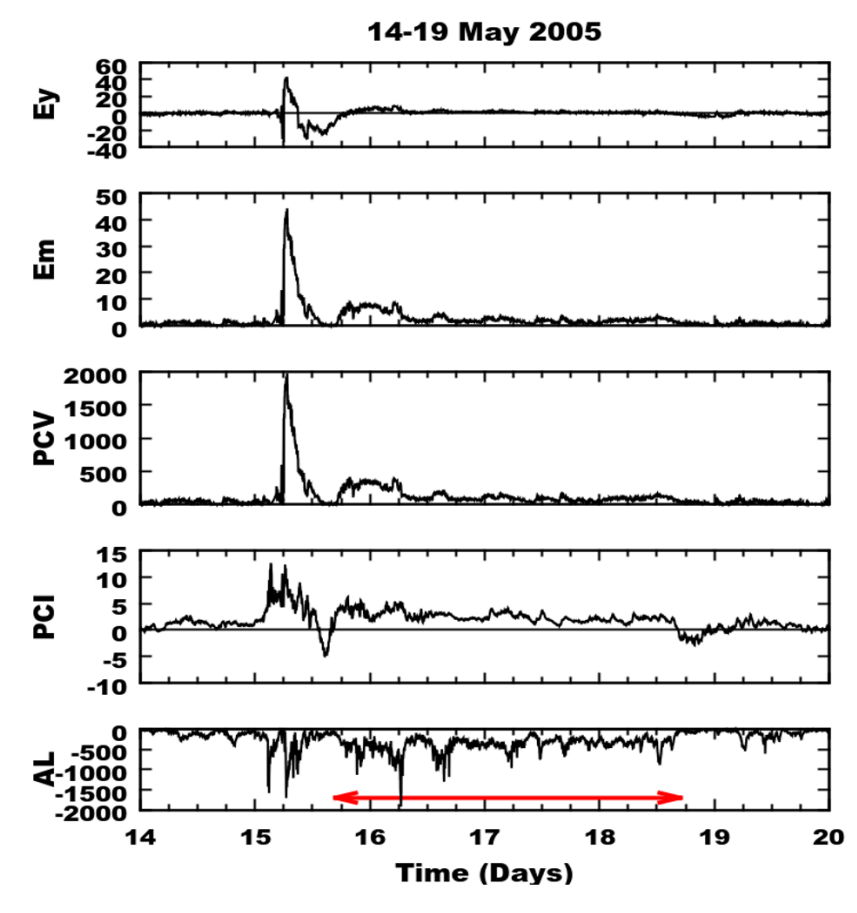

Figure 4. From top to bottom, (a) Interplanetary Electric Field ( $E y$ in $\mathrm{mV} / \mathrm{m}$ ), (b) Polar Cap Potential (PCV in kV), (c) Polar Cap Index $(\mathrm{mV} / \mathrm{m})$ and (d) AL index (nT) for an ICME preceding HILDCAA event during 15-18 May, 2005. The purple horizontal double headed arrow in AL panel represents the HILDCAA interval.

age value of $E y$ for CIR storms is $3.3 \mathrm{mV} / \mathrm{m}$ and the value of Ey studied in this event is also close to this average value. Throughout the HILDCAA event, the value of $E y$ fluctuates around zero. The peak value of PCV observed during the CIR storm is $\sim 400 \mathrm{kV}$ whereas that observed during the HILDCAA event is $\sim 200 \mathrm{kV}$. The PC index has a peak of $\sim 6 \mathrm{mV} / \mathrm{m}$ before the HILDCAA event and $\sim 5 \mathrm{mV} / \mathrm{m}$ during the HILDCAA event. PCI has negative correlation with AL index. During the HILDCAA event, the maximum value of AL is about -1400 nT. According to Moon, [2012], this value indicates intense westward electrojet in auroral ionosphere. The fluctuations in AL index indicates fluctuation in intensity of westward electrojet.

4.1.4. ICME preceding HILDCAA (15th to 18th May 2005). Figure 4 demonstrates the combined plot of variation of $E y$ (in $\mathrm{mV} / \mathrm{m}$ ), $\mathrm{PCV}$ (in $\mathrm{kV}$ ), PCI (in $\mathrm{mV} / \mathrm{m}$ ) and AL (nT) for an ICME preceding HILDCAA event on 15-18 May, 2005. The 

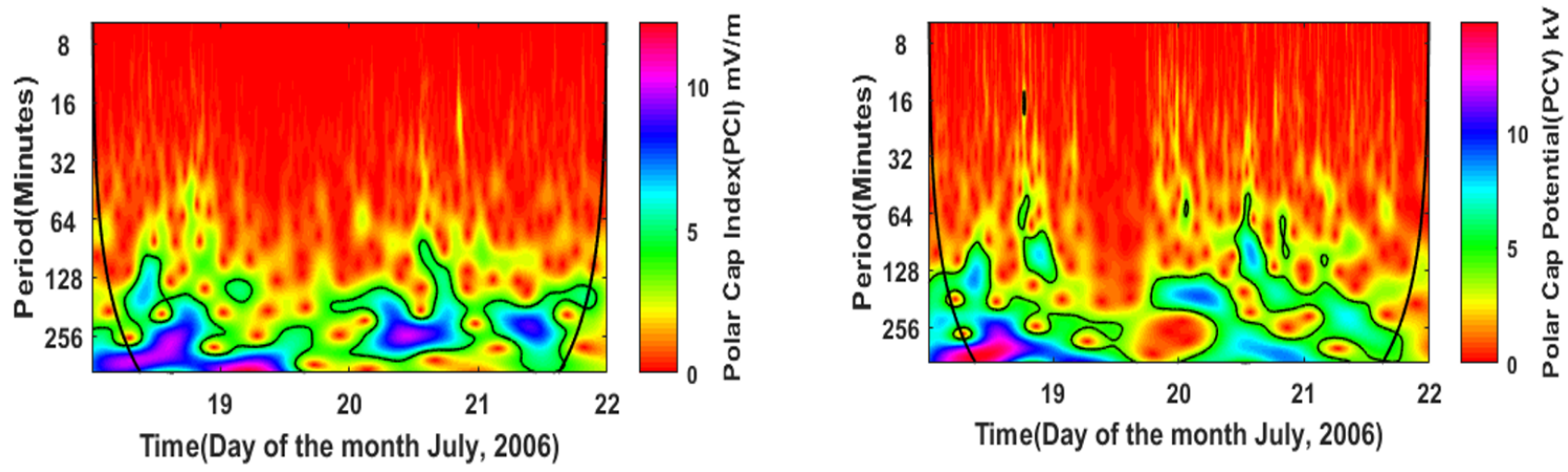

Figure 5. Scalograms for the Wavelet Analysis of the PCI (a) and PCV (b) during the Quiet event of 2006 July 18-21.

ICME storm corresponds to the sharp increase in $E y$, and to PCV index on early morning of 15 th May 2017. Red horizontal double-headed arrow in AL panel of the respective figure signify the HILDCAA period. During the main phase of the ICME storm, i.e., on early morning of 15th May, $E y$ demonstrates east-west perturbation. Its peak value is about $40 \mathrm{mV} / \mathrm{m}$. But during the HILDCAA, it shows almost eastward oscillation. The fluctuation of PCI is comparatively greater than the previous ones for maximum value being $10 \mathrm{mV}$ Figure reveals the intense variability of AL index for a recorded minimum of $-1500 \mathrm{nT}$ with about $-385.44 \mathrm{nT}$ as it's average value during the HILDCAA interval. The result gives clear indications that the HILDCAA time is average of PCV, PCL and AL for the particular event is greater than that of the previous events.

\subsection{Wavelet Analysis}

Figure 5, Figure 6, Figure 7, and Figure 8 represent the scalograms for the PCI (in $\mathrm{mV} / \mathrm{m}$ ) and PCV (in kV) during the quiet event of 18-21 July 2006, non-storm HILDCAA event of 19-24 April 2003, CIR preceding HILCAA of 12-15 February 2004 and ICME preceding HILDCAA event of 1419 May 2005 respectively. The signal power in/ mansities of the PCV and PCI parameters in the wavelet space that are represented on the scalograms are realized using a log2 function that highlights their variations based on various color indices represented. In the figures, the horizontal axis represents the time in days and vertical axis on the left represents the periodicity in minutes. The amplitudes in the plot whose colors
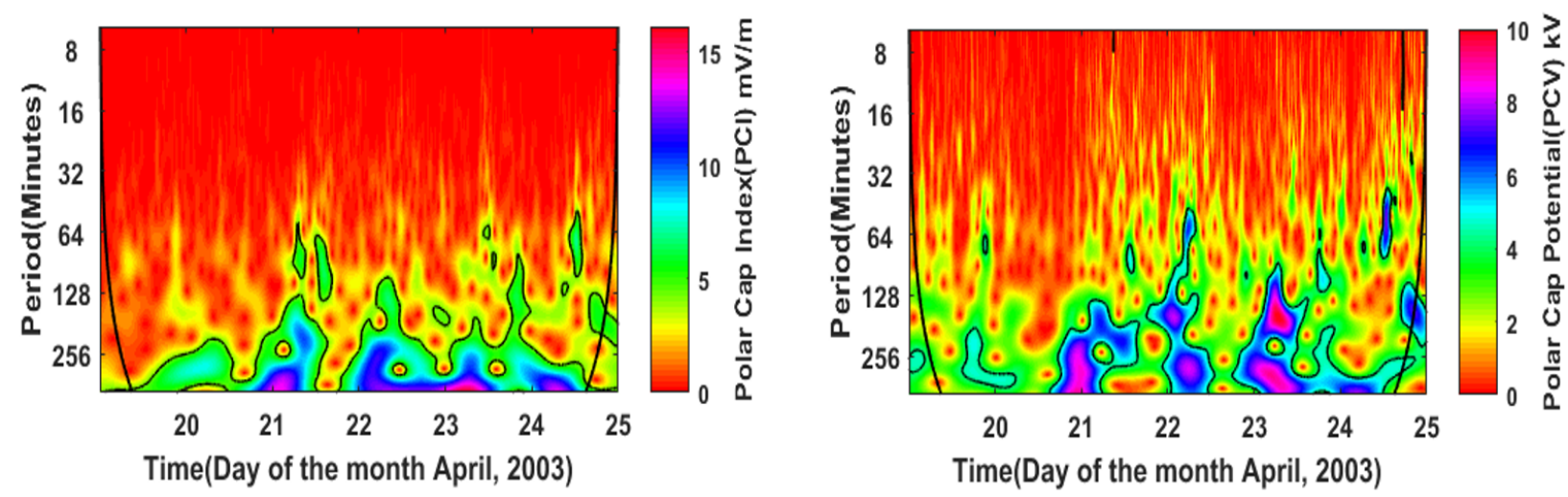

Figure 6. Scalograms for the Wavelet Analysis of the PCI (a) and PCV (b) during the Non-Storm HILDCAA event of 2003 April 19-24. 


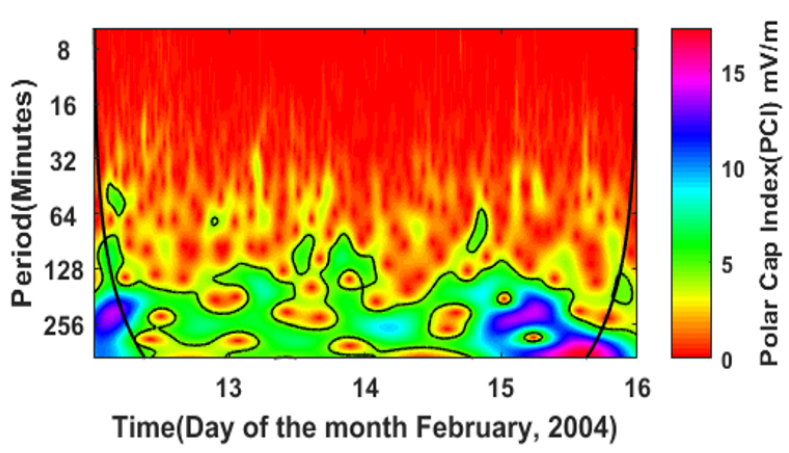

Figure 7. Scalograms for the Wavelet Analysis of the PCI (a) and PCV (b) during the CIR event of 2004 February 12-15.

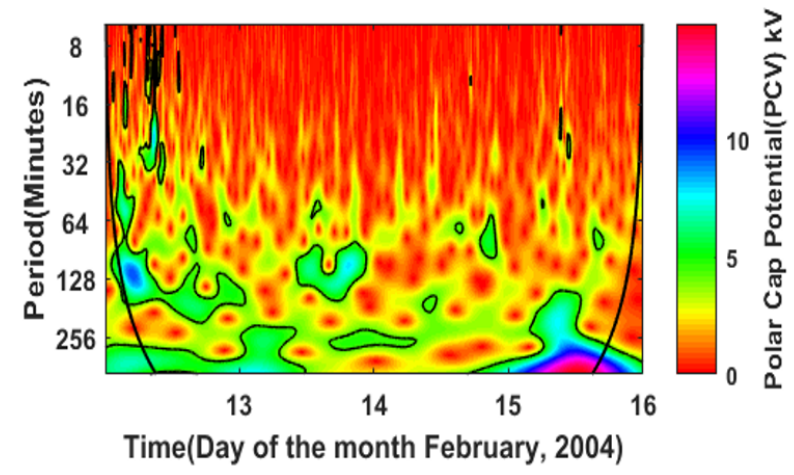

are demonstrated on the right have unit in $\mathrm{kV}$ for $\mathrm{PCV}$ and $\mathrm{mV} / \mathrm{m}$ for PCI. Such unit represents the square estimation of their actual values.

During the Quiet event of 2006 July 18-21, the Polar Cap Index has shown three occurrences of power intensities of amplitude more than 7 units throughout the event with maximum power intensity reaching up to 11 units at the beginning of the July 18. This maximum power intensity lasted throughout the day till the early morning of July 19. However, the occurrences of such power intensities demonstrated a high periodicity of more than 190 minutes. A maximum power intensity has been observed during the mid-day of February 20 that has a periodicity between 150 and 256 minutes. Similar nature has been observed with Polar Cap Potential (PCV) in which a maximum power intensity with maximum periodicity has been observed during the late afternoons of February 18 that lies perfectly inside the cone of significance. Other intensities seemed to vary more abundantly throughout the event days unlike the one of PCI.

The PCI of non storm HILDCAA event of 2003 April 19-24 has the maximum power intensities of up to 14 that occurred on the midnight of April 20, 21 , and 22 and slightly in the early morning hours of April 23. The periodicity of higher power intensities during such occurrences is more than 190 minutes that indicates a lower frequency of occurrence of such intensities during the event. The polar cap potential, however, has shown some very scattered power intensities throughout the event period with peak power intensity reaching up to 10 during the midnight of April 21 and 22. We see very clearly that there is a medium power intensity in timescales more than 50 minutes and higher intensity in time scales more than $150 \mathrm{~min}-$ utes during the early morning hours of the April 23. This clearly indicates that higher power intensities are found in higher time scales whereas lower and medium power intensities are found in
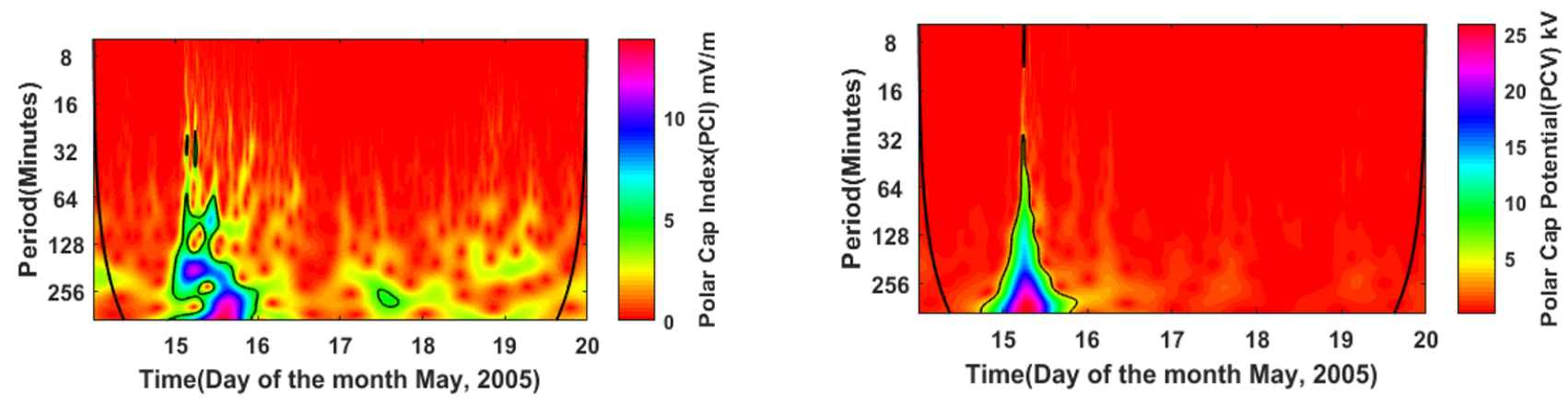

Figure 8. Scalograms for the Wavelet Analysis of the PCI (a) and PCV (b) during the ICME preceding HILDCAA event of 2005 May 14-19. 
lower and medium time scales. The intensities of the PCV are more scattered than that of PCI during this event.

Breaking down the scalograms, the PCI during the CIR event of 2004 February 12-15 has maximum power intensities of up to 20 units that does not lie entirely inside our cone of significance. Most of the power intensities are below 3 units with periodicity of 8-32 minutes. Periodicity greater than 190 minutes is observed for higher power intensities that fit inside our cone of significance. Similar nature has been demonstrated in the PCV. Periodicity of highest power intensities in the PCV is observed to be more than 256 minutes. Unlike PCI, PCV has more scattered power intensities of medium intensity throughout our observed days. In PCI as well as in PCV, higher power intensities are observed in higher time scales whereas medium and lower power intensities are observed in lower and medium time scales.

During the ICME preceding HILDCAA event of 2005 May 14-19, the PCI has shown an instantaneous fluctuation in the power intensities starting the midnight of February 15 that lasted almost the entire day. The maximum power intensities of 12 units had periodicity of more than 150 minutes. However, a notable power intensity of about 7 units has been observed to have a periodicity between 65 to 70 minutes. Rest of the days were rather quiet with a lower value of power intensity almost uniformly distributed throughout the event. The PCV showed a peculiar behavior unlike our previous events as it had almost no variation in power intensities other days except from the late night of May 14 to the night of May 15. A maximum power intensity of more than 20 units was observed during the very early morning of February 15 that had periodicity of more than 240 minutes. This corresponds to the disturbance caused by the ICME storm preceding the HILDCAA event. However, the power intensities of between 10-15 units with periodicity between 65128 minutes were observed which suggests that such power intensities were more frequent during that time. During the HILDCAA period, we observed power intensities from 5 to 10 units which are negligible when compared to the preceding ICME. They have periodicity of more than 48 minutes.

\section{Conclusion}

Amongst the different events selected, the minimum value of PCI and PCV was found during quiet event with an amplitude of $50 \mathrm{kV}$ in PCV and $1 \mathrm{mV} / \mathrm{m}$ in PCI. The highest value of PCI and PCV was found during HILDCAA preceded by ICME with an amplitude of about $500 \mathrm{kV}$ (during HILDCAA) in PCV and $7 \mathrm{mV} / \mathrm{m}$ in PCI. In nonstorm HILDCAA and HILDCAA caused by CIR, the amplitude values of PCV and PCI (during the HILDCAA) were about the same (PCV $=\sim 200 \mathrm{kV}$ and $\mathrm{PCI}=\sim 6 \mathrm{mV} / \mathrm{m}$ ) In this work, we observed the periodicities on PCI and PCV during HILDCAA events. From this study, the results can be summarized as follows:

1. In the Quiet Event, Ey showed very little perturbations. However, during the HILDCAA interval, we observed significant disturbances in $E y$. Even more intense perturbations were found during the storms preceding HILDCAA. Even after the HILDCAA interval on the non-storm HILDCAA event, we see perturbations in Ey. Clearly, the Earth's electric field is perturbed more during HILDCAAs than during Quiet Events. However, we observed more perturbations during non-storm HILDCAA than during CIR-preceded and ICME-preceded HILDCAAs. This indicates that non-storm HILDCAA has a greater impact on earth's electric field than the other two HILDCAAs.

2. Even in PCV and PCI, we see that the nonstorm HILDCAA has perturbations that last even after the HILDCAA interval, possibly due to the reason explained in point 1. Both non-storm and CIR-preceded HILDCAA have similar fluctuations in PCV and PCI. ICME-preceded HILDCAA has a relatively higher value of PCV and PCI fluctuation. However, during the Quiet Event, the perturbations in PCV and PCI are negligible.

3. From wavelet analysis, we found that highest spectral variability occurred in the timescales between 50 and $300 \mathrm{~min}$. (i.e. medium and high timescales). For medium spectral variabilities, we found the timescales to be the same. However, low spectral variabilities were observed across all timescales. Moreover, medium-level spectral variabilities in 
PCV and PCI were continuous in all events except Event 4. In Event 4, we found a high yet discontinuous spectral variability in PCV.

Hence, this study supports that perturbations in PCV and PCI during HILDCAAs have varying frequencies with respect to their intensity. High intensity perturbations are less frequent than the ones with less intensity.

Acknowledgments. The data sets for this study were downloaded from NASA website (https://omniweb. gsfc.nasa.gov/form/omni_min.html). We would like to thank staff members from NASA.

\section{References}

Adhikari, B. (2015), HILDCAA-related effects recorded in middle-low latitude magnetometers, Ph.D. thesis, Instituto Nacional de Pesquisas Espaciais (INPE) Brazil.

Adhikari, B., N. P. Chapagain (2016), Polar cap potential and merging electric field during high intensity long duration continuous auroral activity, Journal of Nepal Physical Society, 3, No. 1, 6-17, https://doi.org/10.3126/jnphyssoc.v3i1.14437

Alves, M. V., E. Echer, W. D. Gonzalez (2006), Geoeffectiveness of corotating interaction regions as measured by Dst index, J. Geophys. Res., 111, A07S05, https://doi.org/10.1029/2005JA011379

Antoine, J. P., R. Murenzi, P. Vandergheynest, S. T. Ali (2008), Two-Dimensional Wavelets and their Relatives, Cambridge University Press, Cambridge.

Boyle, C. B., P. H. Reiff, M. R. Hairston (1997), Empirical polar cap potentials, J. Geophys. Res., 102, 111, https://doi.org/10.1029/96JA01742

Cane, H. V., I. G. Richardson (2003), Interplanetary coronal mass ejections in the near-Earth solar wind during 1996-2002, J. Geophys. Res., 108, 1156, https: / / doi.org/10.1029/2002JA009817

Daubechies, 1. (1992), Ten Lectures on Wavelets, CBMS-NSF Regional Conference (Series in Applied Mathematics), Vol. 61, SIAM, Philadelphia, PA.

Domingues, M. O., O. J. Mendes, A. Mendes da Costa (2005), Wavelet techniques in atmospheric sciences, Adv. Space Res., 35, No. 5, 831-842, https://doi.org/10.1016/j.asr.2005.02.097

Echer, E., B. T. Tsurutani, W. D. Gonzalez (2013), Interplanetary origins of moderate $(-100 \mathrm{nT}<D s t<$ $-50 \mathrm{nT}$ ) geomagnetic storms during solar cycle 23 (1996-2008), J. Geophys. Res. Space Physics, 118, 385392, https://doi.org/10.1029/2012JA018086

Farge, M. (1992), Wavelet transform and their applications to turbulence, Annual Reviews of Fluid Mechanics, 24, 395-457. https://doi.org/10.1146/ annurev.fl.24.010192.002143
Feynman, J., X. Y. Gu (1986), Prediction of geomagnetic activity on time scales of one to ten years, Rev. Geophys., 24, 650-666, https://doi.org/10.1029/ RG024i003p00650

Gonzalez, W. D., et al. (1999), Interplanetary origin of geomagnetic storms, Space Sci. Rev., 88, 529-562, https://doi.org/10.1023/A:1005160129098

Gosling, J. T., V. J. Pizzo (1999), Formation and evolution of corotating interaction regions and their three dimensional structure, Space Sci. Rev., 89, 21, https://doi.org/10.1023/A:1005291711900

Grossmann, A., J. Morlet (1983), Decomposition of Hardy functions into square integrable wavelets of constant shape, SIAM Journal on Mathematical Analysis, 15, 723-73, https://doi.org/10.1137/0515056

Hairston, M. R., K. A. Drake, R. Skoug (2005), Saturation of the ionospheric polar cap potential during the October-November 2003 superstorms, J. Geophys. Res., 110, A09S26, https://doi.org/10.1029/ 2004JA010864

Hajra, R., E. Echer, B. T. Tsurutani, W. D. Gonzalez (2013), Solar cycle dependence of High-Intensity Long-Duration Continuous AE Activity (HILDCAA) events, relativistic electron predictors?, J. Geophys. Res., 118, 5626-5638, https://doi.org/10.1002/jgra. 50530

Hajra, R., et al. (2014), Solar wind-magnetosphere energy coupling efficiency and partitioning: HILDCAAs and preceding CIR storms during solar cycle 23, Journal of Geophysical Research, 119, 2675-2690, https://doi.org/10.1002/2013JA019646

Hubbard, B. B. (1998), The World According to Wavelets: The Story of a Mathematical Technique in the Making, A. K. Peters, Ltd, Natick, MA, USA.

Kamide, Y. (1981), The Relationship between Field-Aligned Current and the Auroral Electrojects: A Review, Space Sci. Rev., 31, No. 2, 127-243, https: / / doi.org/10.1007/BF00215281

Kan, J. R., L. C. Lee (1979), Energy coupling function and solar wind magnetosphere dynamo, Geophysical Research Letters, 6, 577-580, https://doi.org/10.1029 /GL006i007p00577

Klausner, V., et al. (2013), Tsunami effects on the $Z$ component of the geomagnetic field, J. Atmos. Sol. Terr. Phys., 92, 124-136, https://doi.org/10.1016/ j.jastp.2012.10.007

Lau, K. M., H. Y. Weng (1995), Climate signal detection using wavelet transform: How to make a time series sing, Bull. Amer. Meteor. Soc., 76, 2391-2402, https://doi.org/10.1175/1520-0477(1995)076¡2391: CSDUWT ¿2.0.CO;2

Mcelhinny, M. W. (1973), Palaeomagnetism and Plate Tectonics, Cambridge, London.

Miyoshi, Y., et al. (2003), Rebuilding process of the outer radiation belt during the 3 November 1993 magnetic storm: NOAA and Exos-D observations, $J$. Geophys. Res., 108, No. A1, https://doi.org/10. 1029/2001JA007542

Moon, G. H. (2012), Estimation of Polar Cap Potential and the Role of PC Index, Astron. Space Sci., 29, 
No. 3, 259-267, https://doi.org/10.5140/JASS.2012. 29.3.259

Rioul, O., M. Vetterli (1991), Wavelets and signal processing, IEEE Signal Processing Mag., 8, 14-38, https://doi.org/10.1109/79.91217

Ruohoniemi, J. M., K. B. Baker (1998), Largescale imaging of high latitude convection with Super Dual Auroral Radar Network HF radar observation, J. Geophys. Res., 103, 20,797, https://doi.org/10.1029/98 JA01288

Shepherd, G. S. (2006), Polar cap potential saturation: Observations, theory, and modeling, J. Atmos. Sol.Terr. Phys., 69, 234-248, https://doi.org/10.1016/j. jastp.2006.07.022

Smith, E. J., J. H. Wolf (1976), Observation of interaction region and corotating shocks between one and five au: Pioneer 10 and 11, Geophysical Research Letters, 3, 137, https://doi.org/10.1029/GL003i003p0 0137

Søraas, F., et al. (2004), Evidence for particle injection as the cause of Dst reduction during HILDCAA events, J. Atmos. Sol. Terr. Phys., 66, 177-186, https://doi.org/10.1016/j.jastp.2003.05.001

Stewart, J. (1982), Two aspects of meaningful problem solving in science, Sci. Ed., 66, 731-749, https://doi.org/10.1002/sce.3730660509

Tsurutani, B. T., W. D. Gonzalez (1987), The cause of high-intensity long-duration continuous AE activity (HILDCAAs): interplanetary Alfven wave trains, Planet. Space Sci., 35, 405-412, https://doi.org/10. 1016/0032-0633(87)90097-3

Tsurutani, B. T., et al. (2004), Are high-intensity long-duration continuous AE activity (HILDCAA) events substorm expansion events?, J. Atmos. Sol. Terr. Phys., 66, 167-176, https://doi.org/10.1016/j. jastp.2003.08.015

Weimer, D. R. (1996), A flexible, IMF dependent model of high-latitude electric potentials having space weather applications, Geophys. Res. Lett., 23, 2549, https://doi.org/10.1029/96GL02255

Adhikari B., Bhattarai B., Bohara S., Mishra R. K., and Sapkota N., Regmi B. Physics Department, St. Xavier's College, Maitighar, GPO:7437, Kathmandu, Nepal. (binod. adhi@gmail.com)

Narayan P. Chapagain, Department of Physics, Patan Multiple Campus, Lalitpur, Nepal 